Proyecciones Journal of Mathematics

Vol. 37, No 3, pp. 479-489, September 2018.

Universidad Católica del Norte

Antofagasta - Chile

\title{
On multiset group
}

\author{
Binod Chandra Tripathy \\ Tripura University, India
}

Shyamal Debnath

Tripura University, India

and

Debjani Rakshit

Tripura University, India

Received: July 2017. Accepted : November 2017

\begin{abstract}
The concept of multiset is a generalization of Cantor set. In this paper we have attempted to generalize the concept of group in the multiset context and define multiset subgroup and studied some of their basic properties.
\end{abstract}

Key words : Multiset, group, subgroup.

AMS(2010) Classification No: 03E15, 03E20, 03E30, 06F25, $03 B 65,03 B 80$. 


\section{Introduction}

A multiset (mset) is a collection of objects, unlike a standard Cantorian set, in which the elements are allowed to repeat. It is observed from the survey of available literature on multiset and its application that the idea of multiset was hinted by R. Dedikind in 1888 . The multiset theory which contains set theory as a special case was introduced by Cerf et al.[3]. The term multiset, as Knuth noted [5], was first suggested by N.G. de Bruijn in a private communication to him. Further study was carried on by Peterson [7], Yager [9], Blizard ([1], [2]) gave a new dimension to the multiset theory. From a practical point of view multisets are very useful structures arising in many areas of mathematics and computer science. Multiset Topological space has been studied by Shravan and Tripathy [8]. The prime factorization of an integer $n>0$ is a example of a multiset. The terminal string of a non-circular context-free grammer form a multiset which is a set if and only if the grammar is unambiguous.

Research on the multiset theory has not yet gained much ground and it is still in its infant stages. The research carried out so far shows a strong analogy in the behavior of multisets. It is possible to extend some of the main notion and results of sets to the setting of multisets. In 2009, Girish et al. [4] introduced the concepts of relation, function, composition and equivalence in multiset context.

Tella and Daniel [9] have considered set of mappings between multisets and studied about symmetric groups under multiset perspective. Nazmul et.al. [6] have considered the initial universe set to be a group. Then they have defined a group on the multiset derived from the initial universe set. In this article we have considered the general multiset of universal set $X$ and consider $G$, a submset of $X$. Then we define two operations, one on the base set and the other on the multi elements. Then we show that it form a $m$-group. The idea came to us, since different types of groups can be defined from the same universal set. For example, additive, multiplicative groups can be formed on the set of real numbers.

\section{Definitions and Preliminaries}

We procure the following definitions available in the existing literatures, those will be used in this article.

Definition 2.1: A collection of elements which are allowed to repeat is 
called a multiset. Formally if $X$ is a set of elements, a multiset $A$ drawn from the set $X$ is represented by a function $C_{A}$ defined by $C_{A}: X \rightarrow N$, where $N$ represents the set of non negative integers.

For each $x \in X, C_{A}(x)$ is the characteristic value of $x$ in $A$ and indicates the number of occurrences of the element $x$ in $A$. A multiset $A$ is a set if $C_{A}(x)=0$ or 1 for all $x \in X$.

The word "multiset" often used as "mset".

Definition 2.2: Let $A$ be a mset. Then $A^{*}$ is called the root set of $A$ if for each $x$ such that $C_{A}(x)>0$ implies that $x \in A^{*}$, and for every $x$ such that $C_{A}(x)=0$ implies that $x \notin A^{*}$.

i.e, the characteristic function of $A^{*}, C_{A^{*}}(x)= \begin{cases}1, & \text { if } C_{A}(x)>0, \\ 0, & \text { if } C_{A}(x)=0 .\end{cases}$

i.e, $A^{*}$ is an ordinary set.

Definition 2.3: Let $A_{1}$ and $A_{2}$ be two msets drawn from a set $X . A_{1}$ is a sub-mset of $A_{2}\left(A_{1} \subseteq A_{2}\right)$ if $C_{A_{1}}(x) \leq C_{A_{2}}(x) \forall x \in X$. $A_{1}$ is a proper sub-mset of $A_{2}\left(A_{1} \subset A_{2}\right)$ if $C_{A_{1}}(x) \leq C_{A_{2}}(x) \forall x \in X$ and there exists at least one $x \in X$ such that $C_{A_{1}}(x)<C_{A_{2}}(x)$.

Definition 2.4: The cardinality of an mset $A$ drawn from a set $X$ is defined by card $A=\sum_{x \in X} C_{A}(x)$. It is also denoted by $|A|$.

Definition 2.5: The addition of two msets $A_{1}$ and $A_{2}$ drawn from a set $X$ is a mset $A$ denoted by $A=A_{1} \oplus A_{2}$ such that $\forall x \in X, C_{A}(x)=$ $C_{A_{1}}(x)+C_{A_{2}}(x)$.

Definition 2.6: The subtraction of two msets $A_{1}$ and $A_{2}$ drawn from a set $X$ is a mset $A$ denoted by $A=A_{1} \ominus A_{2}$ such that $\forall x \in X, C_{A}(x)=$ $\operatorname{Max}\left\{C_{A_{1}}(x)-C_{A_{2}}(x), 0\right\}$.

Definition 2.7: The union of two msets $A_{1}$ and $A_{2}$ drawn from a set $X$ is a mset $A$ denoted by $A=A_{1} \cup A_{2}$ such that for all $x \in X, C_{A}(x)=$ $\max \left\{C_{A_{1}}(x), C_{A_{2}}(x)\right\}$.

Definition 2.8: The intersection of two msets $A_{1}$ and $A_{2}$ drawn from a set $X$ is a mset $A$ denoted by $A=A_{1} \cap A_{2}$ such that for all $x \in X$, $C_{A}(x)=\min \left\{C_{A_{1}}(x), C_{A_{2}}(x)\right\}$.

Remark 2.1: Let $A$ be a mset from $X$ with $x$ appearing $n$ times in $A$. It 
is denoted by $x \in^{n} A$.

Definition 2.9: Let $A_{1}$ and $A_{2}$ be two msets drawn from a set $X$. Then the Cartesian product of $A_{1}$ and $A_{2}$ is defined by $A_{1} \times A_{2}=\left\{m n /(x, y): x \in^{m} A_{1}, y \in^{n} A_{2}\right\}$.

Throughout the paper, we assume, if $A$ be a mset with the maximum multiplicity $n$, then all the elements of the type $l / x_{1} \in A$ or $x_{1} \in^{l} A$ where $l \leq n$, unless otherwise stated.

\section{Main Results}

We introduce the following definitions:

Definition 3.1: Let $A$ be a non-empty mset whose maximum multiplicity is $n$ and $A^{*}$ be the root set of $A$. Let $m_{1} / x_{1}, m_{2} / x_{2} \in A$. Then ' ${ }^{\prime}{ }^{\prime}$ is called a binary mset composition on $A$ if $m_{1} / x_{1} * m_{2} / x_{2}=m_{1} *_{1} m_{2} / x_{1} *_{2} x_{2}$, where

(i) ${ }^{\prime} *_{1}^{\prime}$ is a binary composition on $N$ and $\left(m_{1} *_{1} m_{2}\right) \leq n$.

(ii) ${ }^{\prime} *_{2}^{\prime}$ is a binary composition on $A^{*}$.

Definition 3.2: Let, ${ }^{\prime}{ }^{\prime}$ is called a binary mset composition on a mset $A$ .Then $A$ is called closed under ' $*$ ' if

$m_{1} / x_{1} * m_{2} / x_{2} \in A$, for all $m_{1} / x_{1}, m_{2} / x_{2} \in A$.

Definition 3.3: A binary mset composition ${ }^{\prime} *^{\prime}$ on a mset $A$ is said to be associative if

$m_{1} / x_{1} *\left(m_{2} / x_{2} * m_{3} / x_{3}\right)$

$=\left(m_{1} / x_{1} * m_{2} / x_{2}\right) * m_{3} / x_{3}$, for all $m_{1} / x_{1}, m_{2} / x_{2}, m_{3} / x_{3} \in A$.

Definition 3.4: A binary mset composition ${ }^{\prime} *^{\prime}$ on a mset $A$ is said to be commutative if

$m_{1} / x_{1} * m_{2} / x_{2}=m_{2} / x_{2} * m_{1} / x_{1}$ for all $m_{1} / x_{1}, m_{2} / x_{2} \in A$.

Definition 3.5: Let $A$ be a mset with maximum multiplicity $n$ and ' $*$ ' be a binary mset composition on $A$. An element $n / e \in A$ is called the identity element of $A$ if

$$
n / e * m / x=m / x=m / x * n / e \text { for all } m / x \in A .
$$


Note 3.1. Without loss of generality, we assume that the identity element appears with the maximum multiplicity in every sub-mset $A$ of $X$.

Example 3.1: Let $\omega$ denote the cube root of unity and $G^{n}=\left[n / 1, n / \omega, n / \omega^{2}\right]$ be a mset with the binary mset composition ${ }^{\prime} *$ defined by

$m_{1} / x_{1} * m_{2} / x_{2}=\min \left\{m_{1}, m_{2}\right\} / x_{1} . x_{2}$, where the binary composition on the root set is the usual multiplication. Then it can be easily verified that in $G^{n}$ the closure property, associative property and commutative property hold.

Definition 3.6: Let $A$ be a mset with maximum multiplicity $n$ and ' $*$ ' be a binary mset composition on $A$. An element $(m / x)^{-1} \in A$ is called the inverse element of $m / x \in A$ if

$$
(m / x)^{-1} * m / x=n / e=m / x *(m / x)^{-1} .
$$

Example 3.2: Let $G^{4}=[4 / 1,4 /-1,4 / i, 4 /-i]$, where $i$ is the imaginary unit, be a mset with the binary composition ${ }^{\prime} *$ is defined by

$$
m_{1} / x_{1} * m_{2} / x_{2}=\ll m_{1}+m_{2} \gg_{n} / x_{1} . x_{2},
$$

where, $\ll m_{1}+m_{2} \gg_{n}=\left\{\begin{array}{cc}m_{1}+m_{2}, & \text { if } m_{1}+m_{2} \leq n \\ \left(m_{1}+m_{2}\right)-n, & \text { if } m_{1}+m_{2}>n\end{array}\right.$

and the binary composition on the root set be the usual multiplication.

Here, $4 / 1$ is the identity element of $G^{4}$, since $4 / 1 * m / x=m / x=$ $m / x * 4 / 1$ for all $m / x \in G^{4}$.

Further every element of $G^{4}$ has a inverse such as $(3 / i)^{-1}=1 /-i$.

Definition 3.7: Let $A$ be a non-empty mset associated with a binary mset composition. Then the algebraic structure $(A, *)$ is called a multi-group of order $n$ if the following axioms are satisfies;

(i) Closure property.

i.e, $m_{1} / x_{1} * m_{2} / x_{2} \in A$, for all $m_{1} / x_{1}, m_{2} / x_{2} \in A$.

(ii) Associative property. 
i.e, $m_{1} / x_{1} *\left(m_{2} / x_{2} * m_{3} / x_{3}\right)=\left(m_{1} / x_{1} * m_{2} / x_{2}\right) * m_{3} / x_{3}$, for all $m_{1} / x_{1}$, $m_{2} / x_{2}, m_{3} / x_{3} \in A$.

(iii) Existence of identity.

i.e, $n / e * m / x=m / x=m / x * n / e$ for all $m / x \in A$.

(iv) Existence of inverse.

i.e, there exists a $(m / x)^{-1} \in A$ for all $m / x$ such that $(m / x)^{-1} * m / x=$ $n / e=m / x *(m / x)^{-1}$.

Remark 3.1: The mset $G^{4}$ of Example 3.2 forms a multi-group of order 4 under the binary composition ' $*$ ' defined by

$m_{1} / x_{1} * m_{2} / x_{2}=\ll m_{1}+m_{2} \gg_{n} / x_{1} \cdot x_{2}$.

Theorem 3.1: In a multi-group, the identity element is unique.

Proof: Let $(A, *)$ be a multi-group of order $n$.

Suppose that $n / e$ and $n / e^{\prime}$ be two identity elements of $A$.

Then from above definition we have,

$n / e * n / e^{\prime}=n / e^{\prime}$ and $n / e * n / e^{\prime}=n / e$.

i.e, $n / e=n / e^{\prime}$.

Theorem 3.2: For each element in a multi-group, there is an unique inverse element.

Proof: Let $(A, *)$ be a multi-group of order $n$ and $m / a \in A$.

Suppose that $m_{1} / b$ and $m_{2} / c$ be two inverses of $m / a$.

Then by the above definition we have, 


$$
\begin{aligned}
& m / a * m_{1} / b=m_{1} / b * m / a=n / e \\
& \text { and } m / a * m_{2} / c=m_{2} / c * m / a=n / e . \\
& \text { Now, } m_{1} / b=m_{1} / b * n / e \\
& =m_{1} / b *\left(m / a * m_{2} / c\right) \\
& =\left(m_{1} / b * m / a\right) * m_{2} / c \\
& =n / e * m_{2} / c \\
& =m_{2} / c .
\end{aligned}
$$

Hence the proof.

Definition 3.8: Let $(A, *)$ be a multi-group. Then the multi-group is called an abelian multi-group if it satisfies the commutative property.

Definition 3.9: A multi-subgroup of a multi-group $(A, *)$ is a sub-mset of $A$ which is also a group with respect to the same binary mset composition ' $*$ ' as in $A$.

Note 3.1. If $(A, *)$ be a multi-group of order $n$ then $A$ and $[n / e]$ be two trivial multi-subgroups of $A$.

Example 3.3: The multiset group discussed in Example 3.2, i.e. $\left(G^{4}, *\right)$ is an abelian multi-group.

Example 3.4: Consider the mset group $G^{4}$ of Example 3.2, let $S^{4}=$ $[4 / 1,4 /-1]$ is a non trivial multi-subgroup of $G^{4}$.

Remark 3.3: $S$ be a multi-subgroup of $A$, if

(i) $S^{*}$ be a subgroup of $A^{*}$.

(ii) $S$ has the same maximum multiplicity as of $A$.

Theorem 3.3: A necessary and sufficient condition for a non-empty sub- 
mset $S$ of a multi-group $(A, *)$ of order $n$ to be a multi-subgroup is that for all $m_{1} / a, m_{2} / b \in S$ implies $m_{1} / a *\left(m_{2} / b\right)^{-1} \in S$.

Proof: Assume that $S \neq \emptyset$ and $m_{1} / a *\left(m_{2} / b\right)^{-1} \in S$, for all $m_{1} / a, m_{2} / b \in$ $S$.

Now if we take, $m_{2} / b=m_{1} / a$,

then $m_{1} / a *\left(m_{1} / a\right)^{-1}=n / e \in S$ [Existence of identity].

Since $n / e \in S$, for any $m_{1} / a \in S$,

$n / e *\left(m_{1} / a\right)^{-1}=\left(m_{1} / a\right)^{-1} \in S$ [Existence of inverse].

Now for any $m_{1} / a, m_{2} / b \in S$ which also implies $\left(m_{2} / b\right)^{-1} \in S$, $m_{1} / a *\left(\left(m_{2} / b\right)^{-1}\right)^{-1}=m_{1} / a * m_{2} / b \in S$ [Closure property].

Also the associative property holds in $S$, since $S$ is a sub-mset of $A$ i.e, $S$ is a multi-subgroup.

Conversely, let $S$ be a of a multi-subgroup of a multi-group $A$ of order $n$.

Then $n / e \in S$, i.e, $S \neq \emptyset$.

If $m_{1} / a, m_{2} / b \in S$ then $\left(m_{2} / b\right)^{-1} \in S$.

So by closure property we have, $m_{1} / a *\left(m_{2} / b\right)^{-1} \in S$.

Theorem 3.4: Intersection of two multi-subgroups is again a multi-subgroup.

Proof: Let $S_{1}$ and $S_{2}$ be two multi-subgroups of a multi-group $A$ of order $n$.

Then $S_{1} \cap S_{2} \neq \emptyset$, since $n / e \in S_{1} \cap S_{2}$.

Let, $m_{1} / a, m_{2} / b \in S_{1} \cap S_{2}$.

Therefore $m_{1} / a, m_{2} / b \in S_{1}$ and also $m_{1} / a, m_{2} / b \in S_{2}$. 
So by Theorem 3.3 we have $m_{1} / a *\left(m_{2} / b\right)^{-1} \in S_{1}$ and also $m_{1} / a *$ $\left(m_{2} / b\right)^{-1} \in S_{2}$ i.e, $m_{1} / a *\left(m_{2} / b\right)^{-1} \in S_{1} \cap S_{2}$.

Hence the theorem.

Result 3.5: The union of two multi-subgroups may not be a multi-subgroup.

Example 3.5: Let, $(A, *)$ be a multi-group of order $n$ where the root set $A^{*}=\{0, \pm 1, \pm 2, \ldots \ldots\}$ and the binary composition ${ }^{\prime} *^{\prime}$ defined by

$$
m_{1} / x_{1} * m_{2} / x_{2}=\ll m_{1}+m_{2} \gg_{n} / x_{1}+x_{2},
$$

where, $\ll m_{1}+m_{2} \gg_{n}=\left\{\begin{array}{cc}m_{1}+m_{2}, & \text { if } m_{1}+m_{2} \leq n \\ \left(m_{1}+m_{2}\right)-n, & \text { if } m_{1}+m_{2}>n\end{array}\right.$ and the binary composition on the root set is the usual addition.

Here $S_{1}=[\ldots, n /-4, n /-2, n / 0, n / 2, n / 4, \ldots]$

and $S_{2}=[\ldots, n /-6, n /-3, n / 0, n / 3, n / 6, \ldots]$

are two multi-subgroups of $A$.

Now $S_{1} \cup S_{2}=[\ldots, n /-3, n /-2, n / 0, n / 2, n / 3, \ldots]$ which is not closed.

Since, $n /-3 * n /-2 \notin S_{1} \cup S_{2}$.

\section{References}

[1] Blizard W D., Multiset Theory, Notre Dame Jour. logic, 31, pp. 36-65, (1989).

[2] Blizard W. D., The development of multiset theory, Modern Logic, 1 (4), pp. 319-352, (1991).

[3] Cerf V., Fernandez E., Gostelow K. and Volausky S., Formal control and low properties of a model of computation, Report ENG 7178, 
Computer Science Department, University of California, Los Angeles, CA, December, pp. 81, (1971).

[4] Girish K. P. and John S. J. Relations and functions in multiset context, Inf. Sci., 179, pp. 758-768, (2009).

[5] Knuth D. E. The Art of computer programming, Vol. 2 (Seminumerical Algorithms), 2nd ed. Addison-Wesley, (1981), Reading Mass.

[6] Nazmul S. K., Majumdar P. and Samanta S. K., On multisets and multigroups, Anals Fuzzy Math. Inf., 6 (3), pp. 643-656, (2013).

[7] Peterson J. Computation sequence sets, Jour. Computer Syst. Sci., 13 (1), pp. 1-24, (1976).

[8] Shravan K. and Tripathy B. C., Generalised closed Sets in multiset topological space, Proyecciones Jour. Math. Vol. 37, 2, pp. 223-237, (2018) .

[9] Tella Y. and Daniel S., A Study of Group Theory in the Context of Multiset Theory. Internat. Jour. Sci. Tech., 2 (8), pp. 609-615, (2013).

[10] Yager R. R. On the theory of bags. Internat. Jour. Gen. Syst., 13, pp. 23-37, (1986).

\author{
Binod Chandra Tripathy \\ Department of Mathematics, \\ Tripura University, \\ Agartala - 799022, \\ Tripura, \\ India \\ e-mail : tripathybc@rediffmail.com
}

\title{
Shyamal Debnath
}

Department of Mathematics,

Tripura University,

Agartala - 799022,

Tripura,

India

e-mail : shyamalnitamath@gmail.com

and 
Debjani Rakshit

Department of Mathematics,

Tripura University,

Agartala - 799022,

Tripura,

India

e-mail : debjanirakshit88@gmail.com 